\title{
6-Monoacetylmorphine Measurement
}

National Cancer Institute

\section{Source}

National Cancer Institute. 6-Monoacetylmorphine Measurement. NCI Thesaurus. Code C74876.

The determination of the amount of 6-monoacetylmorphine present in a sample. 